MRS Advances (C) 2017 Materials Research Society

DOI: $10.1557 /$ adv.2017.89

\title{
Focused Ion Beam Fabrication of SU-8 Waveguide Structures on Oxidized Silicon
}

\author{
Swagata Samanta ${ }^{1}$, Pallab Banerji ${ }^{2}$ and Pranabendu Ganguly ${ }^{1}$ \\ ${ }^{1}$ Advanced Technology Development Centre, Indian Institute of Technology Kharagpur, \\ West Bengal, Kharagpur 721302 , India. \\ ${ }^{2}$ Materials Science Centre, Indian Institute of Technology Kharagpur, \\ West Bengal, Kharagpur 721302, India.
}

\begin{abstract}
This work deals with SU-8 waveguides and waveguide structures fabricated on an oxidized silicon substrate using 'Focused ion beam (FIB) lithography'. From our experimentation it seems that FIB method is practically not suitable for fabricating long SU-8 waveguide structures, rather it is more suitable for nanoscale modification of already fabricated waveguides, such as, to fabricate photonic crystal structures.
\end{abstract}

\section{INTRODUCTION}

Conventional UV lithography has been the mainstay for fabrication of micro-devices for decades. High resolution, which is required for more compact nano-devices, can be somewhat obtained with the expense of depth of focus, hence the device designs involve a tradeoff between short wavelength and large numerical apertures [1-3].The limitation of resolution due to diffraction results in searching alternative lithography techniques, which are being developed in research laboratories throughout the world. Moreover, dependency on masks complicates the fabrication as a new mask must be generated for each new design. Generating a new mask is neither cost nor time efficient. Different mask-less lithography techniques are developed whose key advantage is the ability to change lithography patterns from one run to the next, without incurring the cost of generating a new photomask. Some of the maskless techniques include electron beam lithography (EBL), focused ion beam (FIB) lithography, scanning probe lithography (SPL), direct laser writing (DLW) technique, and dip pen lithography (DPL) [4-8]. Optical waveguides and devices using SU-8 material are of today's interest due to their potential applications in optical communications, optical interconnect, and integrated optics. SU-8 exhibits a huge potential for micro-technologies as it can be spin coated at a thickness ranging from a millimeter to a few microns and it gives very high aspect ratios when patterned $[9,10]$. SU-8 being a low cost material and optically transparent in the visible region as well as at telecommunication wavelengths of $1.3-1.6 \mu \mathrm{m}$ makes it a suitable material for optical waveguides. Both single- and multi-mode waveguides using SU-8 were fabricated before by many research groups with variations in cladding material (air, polymers) using different fabrication techniques (both mask and maskless approaches) $[8,11,12]$.

In this work, we have used maskless focused ion beam lithography, where focused beam of ions $\left(\mathrm{Ga}^{+}\right)$hit the sample surface and mills the required portion of material to create air-cladded SU-8 waveguide structures. To the best of our knowledge, this is the first attempt to fabricate SU-8 polymer waveguide structures by using FIB technique. The limitations of using this method on the SU-8 polymer are also discussed and we have highlighted some of the fabricated structures for fast prototyping of photonic applications. 


\section{SAMPLE PREPARATION AND FABRICATION}

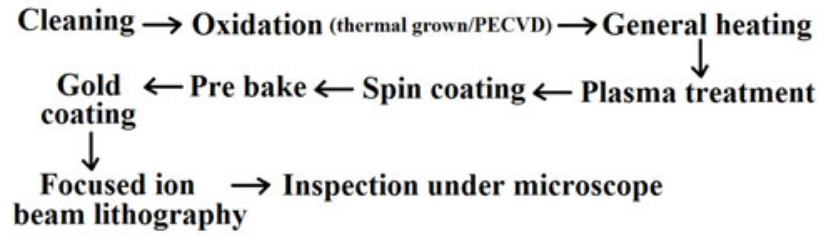

Fig.1 Flow diagram of sample preparation and fabrication

Fig. 1 shows the flow diagram of sample preparation and fabrication. Our starting material is silicon (100) substrate. First of all, the sample is cleaned by well-known Piranha cleaning; hydrogen peroxide $\left(\mathrm{H}_{2} \mathrm{O}_{2}\right)$ is poured in a quartz beaker and concentrated sulphuric acid $\left(\mathrm{H}_{2} \mathrm{SO}_{4}\right)$ is slowly dropped into it (volume ratio of the mixture is $1: 1$ ), and left undisturbed for $25 \mathrm{mins}$. Next, it is thoroughly rinsed with de-ionized (DI) water having resistivity $18.2 \mathrm{M} \Omega$ $\mathrm{cm}$ and is blown with dry nitrogen jet and is put in oxidation furnace. After reaching a temperature of $1050^{\circ} \mathrm{C}$, it is oxidized in a sequence of dry-wet-dry for $30 \mathrm{mins}-2 \mathrm{hrs}-30 \mathrm{mins}$ respectively to obtain an oxide thickness of $1 \mu \mathrm{m}$, which is uniform throughout the wafer surface. Then it has undergone a general heating for $30 \mathrm{mins}$ at $150^{\circ} \mathrm{C}$. Oxygen Plasma treatment is carried out at a power of $40 \mathrm{~W}$ for $45 \mathrm{secs}$. It is then spin coated with SU-8 5 polymer at $500 \mathrm{rpm}$ for $10 \mathrm{secs}$ and ramped to $6000 \mathrm{rpm}$ for next 20 secs to achieve a resist thickness of $1.5 \mu \mathrm{m}$ (measured by Dektak surface profiler). It is then baked on a horizontal hotplate at $65^{\circ} \mathrm{C}$ and $95^{\circ} \mathrm{C}$ for $1 \mathrm{~min}$ and 3 mins respectively, and cooled at room temperature for 10mins for thermal relaxation. Then the sample is placed on Quorum DC sputtering goldpalladium (80:20\%) coater for about 2 mins before proceeding to focused ion beam milling (using AURIGIA Compact Zeiss FIB machine). A high resolution of $30 \mathrm{keV} \mathrm{Ga}^{+}$ions in combination with a high-precision field emission gun is used for proper positioning and inspection of the fabricated structures. The pattern drawn in standard GDSII format (using Elphy Quantum software, Raith) consists of five parallel straight waveguides of varying widths and is written directly on the wafer surface with a pattern generator; the writing method being raster scanning in dot sequence. Fig. 2 shows the description method for FIB apparatus. The stage with sample is tilted $54^{0}$ so that it becomes perpendicular to the ion gun, which is necessary for ion milling. The working distance for simultaneously milling and imaging (coincidence point) is $5 \mathrm{~mm}$.

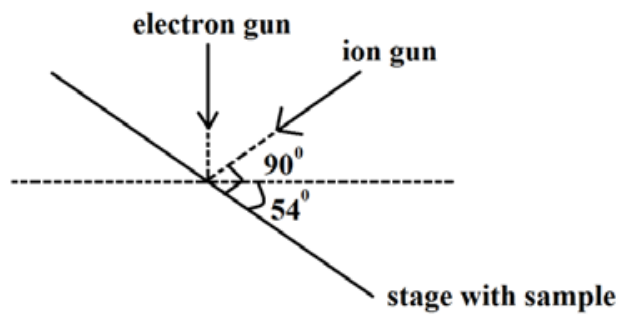

Fig. 2 Description method for FIB apparatus 


\section{RESULTS AND DISCUSSIONS}

The maximum writing field for AURIGIA Compact FIB machine is $197 \mu \mathrm{m}^{2}$; beyond that area stitching is required, which has a tolerance of $\pm 0.5 \mu \mathrm{m}$. Also, the minimum spot size that we may get is $30 \mathrm{~nm}$ with a minimum current of $1 \mathrm{pA}$. In order to make SU-8 applicable for waveguide purpose, that means, for proper optical characterization, patterns should be fabricated edge-to edge of the wafer. Moreover, for easy handling of sample, the sample length should be atleast $3-4 \mathrm{~mm}$. Now, to scan a sample which is of $3-4 \mathrm{~mm}$ length with small ion dose will take huge amount of time which is practically not feasible. Due to the heavy mass of $\mathrm{Ga}+$ ions, interaction of gallium beam with the sample surface is destructive in nature. Also, the high energy ion bombardment on the sample surface may gradually gather several volts of charge resulting in cavity or hole, and local melting due to electrostatic discharge. Looking on the limitations, we proceed with a beam current of $10 \mathrm{nA}$, which is kept fixed (beam shape being circular and a beam diameter or spot size of $1.5 \mu \mathrm{m}$ ), and ion doses are varied. The milling depth is adjusted by this ion dose (that is, the time for which the ion stays on each spot while milling). The width of the waveguides as inspected under FESEM does not match as drawn in the pattern. The widths are broadened due to the application of high beam current of 10nA (depicted in Fig. 3), as FIB has a drawback of backscattering and redeposition while milling of structures. Actually, when we are increasing the ion dose to achieve more depth, the staying of ion beam on a spot also increases by the time the dose is increased; thus, automatically the width will be broadened. The milling depths as obtained from atomic force microscopy (AFM) for 1, 15 and 40 ion doses are $0.195 \mu \mathrm{m}, 0.34 \mu \mathrm{m}$ and $0.57 \mu \mathrm{m}$ respectively (as depicted in Table 1 ). The root-mean-square (rms) roughness values are $0.118 \mu \mathrm{m}, 0.415 \mu \mathrm{m}$ and $0.512 \mu \mathrm{m}$ for 1,15 and 40 ion doses respectively. Now, the permissible roughness is one-tenth of the transmitting wavelength $(1.55 \mu \mathrm{m})$, above which the scattering loss will be high; thus, ion dose 1 with roughness $0.118 \mu \mathrm{m}$ is only acceptable for waveguide purpose.

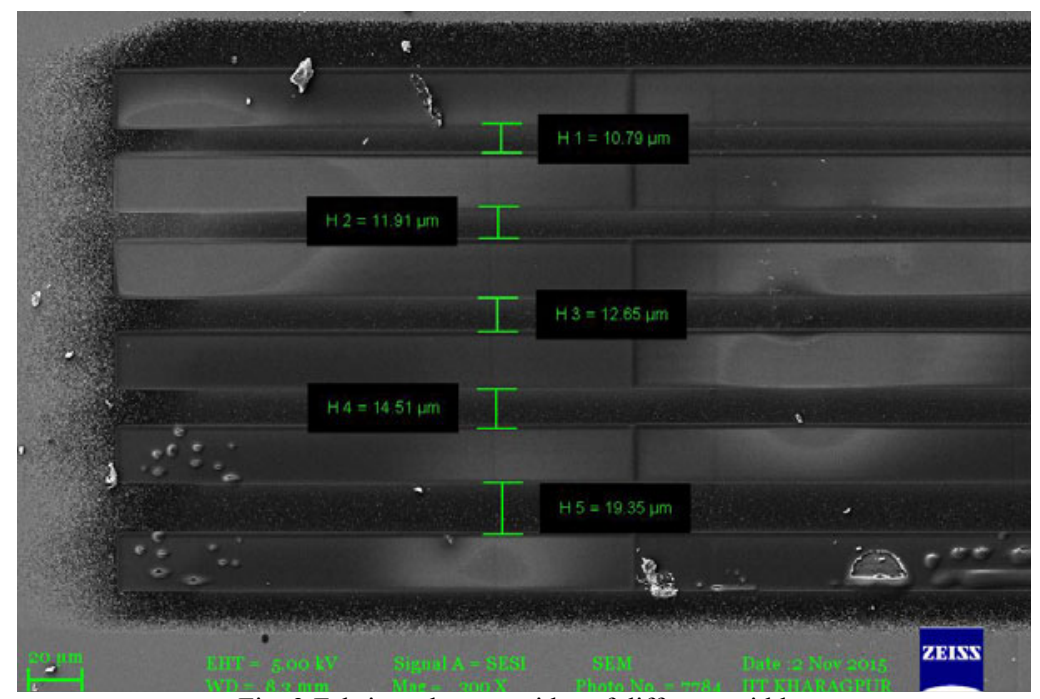

Fig. 3 Fabricated waveguides of different widths 
However, the milling depth that we achieve with this ion dose 1 is only $0.195 \mu \mathrm{m}$. In order to obtain more depth, the ion dose is to be increased; but at the same time there is an increase in the surface roughness as well, which means more prone to optical losses. Thus, we find that FIB method is practically not suitable for fabricating long SU-8 waveguide structures.

Table 1 Topography of waveguides for different ion doses and corresponding milling depth

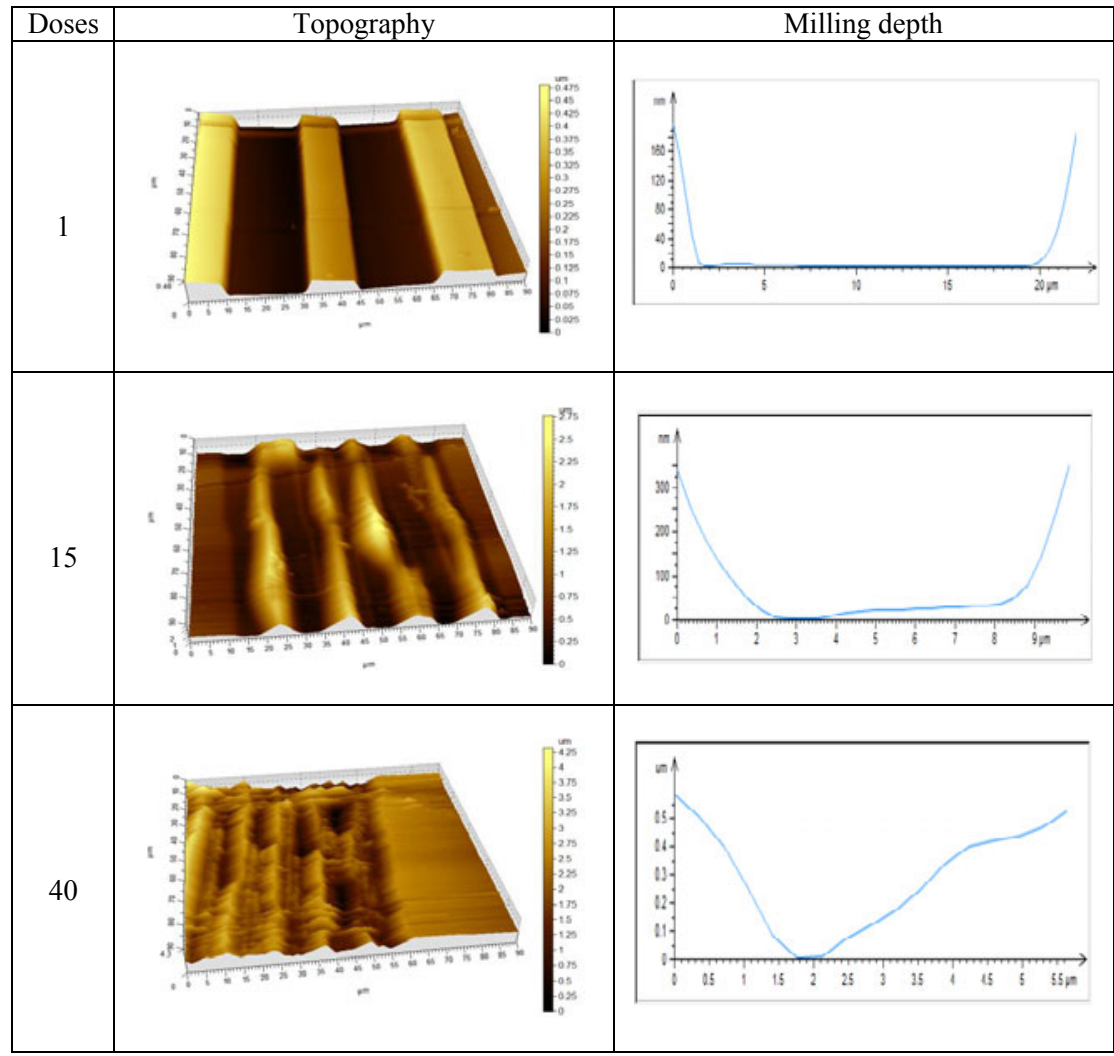

Fig.4 shows the scanning electron micrograph (SEM) of the waveguide edge where the dose for FIB lithography is taken as 15 . It can be seen from the figure that the portion of the silicon wafer where there is no SU-8 coating on it, the waveguide pattern is fair, and thus we may say that FIB lithography is favorable on silicon rather than SU-8 polymer at this particular dose. 


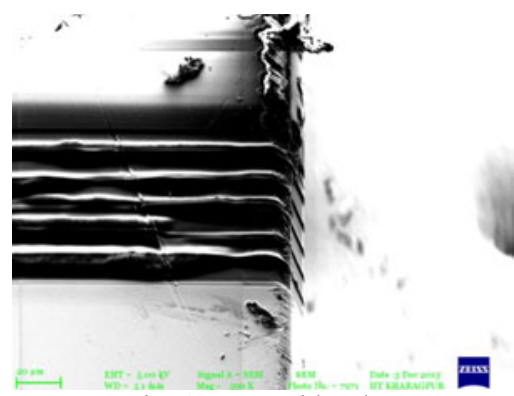

Fig. 4 Waveguide edge

However, FIB lithography on SU-8 can be successfully applied in fabricating photonic crystals and making any minor changes in micro- or nano-structures. Fig. 5 shows our fabricated photonic crystal waveguide. Here we have used plasma enhanced chemical vapor system (PECVD) to deposit $\mathrm{SiO}_{2}$ on silicon sample, on which $\mathrm{SU}-8$ is coated, and by using FIB photonic crystal waveguide is fabricated. Each hole of the photonic crystal waveguide is of $\sim 460 \mathrm{~nm}$ in diameter and the periodicity is $\sim 970 \mathrm{~nm}$. The technique may be useful to fabricate different photonic crystal structure based SU-8 waveguide devices, such as, generation of slow light, optical filters, low-loss $90^{\circ}$-bent waveguides, etc.
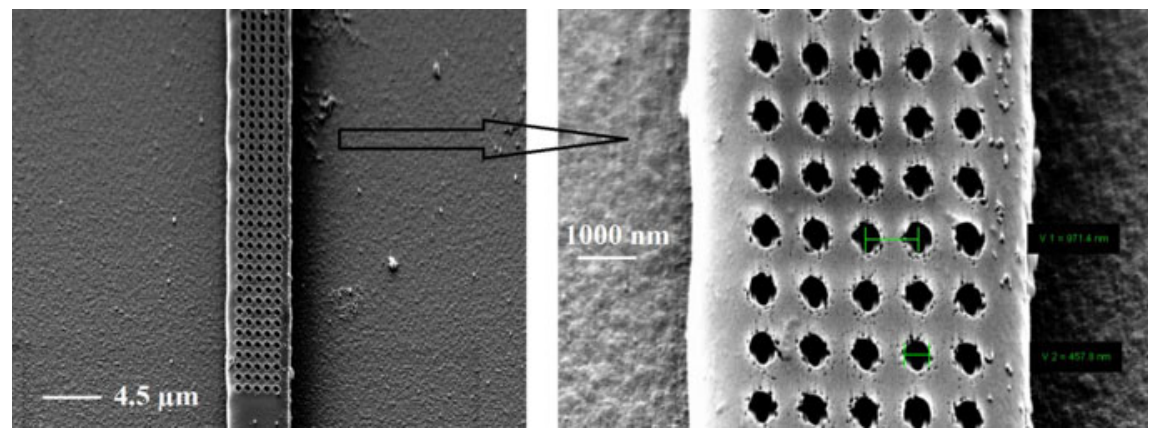

Fig. 5 Photonic crystal waveguide

Fig.6 shows one of the much required modifications to fabricate two-waveguide optical directional coupler with very narrow $150 \mathrm{~nm}$ separation between the waveguides in the coupling region. By conventional photolithography, this $150 \mathrm{~nm}$ feature size is impossible to achieve due to the diffraction limit. Since FIB is not suitable for long structures, in the first step the directional coupler was fabricated by conventional UV-photolithography. The structure what we have obtained was a zero-gap coupler, having no separation between the waveguides in the coupled region. Then in the second step FIB is applied to make a groove of $150 \mathrm{~nm}$ width, well-aligned with the waveguides, throughout the centre of coupled region of the directional coupler. This kind of devices are the building blocks of many integrated optic devices, such as, power splitters, micro-ring resonators etc., where coupling coefficient between the waveguides can be precisely controlled by changing the groove width very precisely, which is beyond the scope of conventional UV photolithography. 

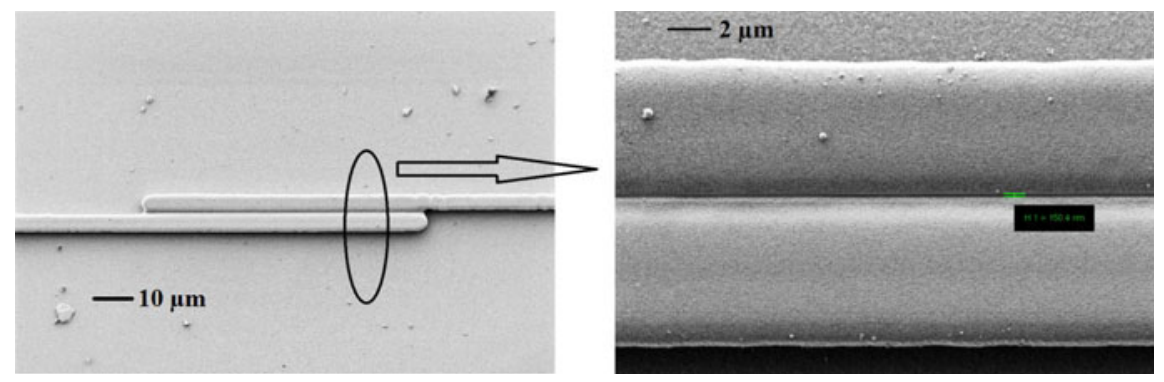

Fig. 6 Two-waveguide optical coupler with $150 \mathrm{~nm}$ separation in between the waveguides

\section{CONCLUSIONS}

Focused ion beam (FIB) lithography requires proper beam size, shape, and a well control of ion current and dose in order to mill the required portion of material, so that the sample surface won't be rough which will result in optical loss; and low optical loss is an important requirement for any waveguide fabrication technique. Though FIB is an effective technique in rapid fabrication of several prototype devices, it is not favorable for fabricating long conventional SU-8 waveguides. However, it is well-suited in fabricating photonic crystal structures or making any precise modifications in micro- and nanometer photonic structures. Reported fabricated structures in this work are our first experimental results, which can obviously be improved by process optimization and detail optical characterization.

\section{ACKNOWLEDGMENT}

We would like to thank Prof. Jatindra Nath Roy, Dr. Manish K. Hooda, Mr. Pradip K. Dey and Mr. Nilay Bhowmik for their help and support during the experimentation.

\section{REFERENCES}

1. R. Lawes, Applied Surface Science 36 (1-4), 485-489 (1989).

2. F. Demami, L. Pichon, R. Rogel and A. Salaün, IOP Conference Series: Materials Science and Engineering 6 (2009).

3. C. Lin, G. Lee, B. Chang and G. Chang, J. Micromechanics and Microengineering 12 (5), 590-597 (2002).

4. B. Bilenberga, S. Jacobsena, M. S. Schmidta, L. H. D. Skjoldinga, P. Shib, P. Boggilda, J. O. Tegenfeldtc and A. Kristensena, Microelectronic Engineering 83 (49), 1609-1612 (2006).

5. A. K. Nallani, S. W. Park and J. B. Lee, Proc. SPIE, 5116 (2003).

6. K. Gamo, Nuclear Instruments and Methods in Physics Research B65, 40-49 (1992).

7. C. Acikgoz, M. A. Hempenius, J. Huskens and G. J. Vancso, J. European Polymer 47 (11), 2033-2052 (2011).

8. S. Samanta, P. K. Dey, P. Banerji and P. Ganguly, Optics Commun. 382 (2016).

9. J. Lee, K. Choi, and K. Yoo, Micromachines 6 (1), 1-18 (2014).

10. B. Yang, L. Yang, R. Hu, Z. Sheng, D. Dai, Q. Liu and S. He, J. Lightwv. Technol. 27 (18), 4091-4096 (2009).

11. M. Nordstrom, D. Zauner, A. Boisen and J. Hubner J. Lightwv. Technol. 25 (5), 1284-1289 (2007).

12. P. K. Dey and P. Ganguly, J. Optics 43 (1), 73-79 (2013). 